\title{
The clinical effectiveness of permissive hypotension in blunt abdominal trauma with hemorrhagic shock but without head or spine injuries or burns: a systematic review
}

\author{
This article was published in the following Dove Press journal: \\ Open Access Emergency Medicine \\ 30 May 2012 \\ Number of times this article has been viewed
}

\author{
Abdulrahman Alsawadi \\ Colchester Hospital University NHS \\ Foundation Trust, Colchester, \\ Essex, United Kingdom
}

Background: Trauma is a major cause of death and disability. The current trend in trauma management is the rapid administration of fluid as per the Advanced Trauma Life Support guidelines, although there is no evidence to support this and even some to suggest it might be harmful. Some guidelines, protocols, and recommendations have been established for the use of permissive hypotension although there is reluctance concerning its application in blunt injuries.

Objectives: The aim of this review is to determine whether there is evidence of the use of permissive hypotension in the management of hemorrhagic shock in blunt trauma patients. This review also aims to search for any reason for the reluctance to apply permissive hypotension in blunt injuries. Methods: This systematic review has followed the steps recommended in the Cochrane Handbook for Systematic Reviews of Interventions. It is also being reported in accordance with the Preferred Reporting Items for Systematic Reviews and Meta-Analyses Statement and checklist. Database searches of MEDLINE, EMBASE, the Centre for Reviews and Dissemination databases and the Cochrane Library were made for eligible studies as well as journal searches. Inclusion criteria included systematic reviews that have similar primary questions to this review and randomized controlled trials where patients with blunt torso injuries and hemorrhagic shock were not excluded. Rapid or early fluid administration was compared with controlled or delayed fluid resuscitation and a significant outcome was obtained.

Results: No systematic reviews attempting to answer similar questions were found. Two randomized controlled trials with mixed types of injuries in the included patients found no significant difference between the groups used in each study. Data concerning the question of this review was sought after these papers were appraised.

Conclusion: The limited available data are not conclusive. However, the supportive theoretical concept and laboratory evidence do not show any reason for treating blunt injuries differently from other traumatic injuries. Moreover, permissive hypotension is being used for some nontraumatic causes of hemorrhagic shock and in theater. Therefore, this should encourage interested researchers to continue clinical work in this important field.

Keywords: trauma management, fluid administration, ATLS, permissive hypotension, hypotensive resuscitation

\section{Introduction}

\section{Rationale}

The control of bleeding is a widely agreed concept in the management of hemorrhagic shock. ${ }^{1,2}$ However, no consensus has yet been reached on the best specific form of 
management to be used from the time of the trauma until bleeding control is achieved. Several strategies and protocols have been suggested and studied, but no strong evidence for their efficacy has yet been provided. ${ }^{3-5}$ Nevertheless, it has been routine practice to start rapid fluid administration during the early management of trauma in patients. ${ }^{3,6,7}$ This was an especially common practice during the second half of the twentieth century under the Advanced Trauma Life Support (ATLS) guidelines ${ }^{1,8}$ and continues to be the current recommended approach in ATLS courses. ${ }^{2}$

The concept of aggressive fluid resuscitation is aimed at normalizing the patient's vital signs as quickly as possible $e^{2,7,9,10}$ and at correcting the depletion in intravascular volume that causes inadequate tissue perfusion. ${ }^{2,11-13}$ However, this approach has been widely questioned by many authors over the last two decades as it is not supported by enough evidence. Moreover, the models on which this method is based do not represent the management of trauma victims, since bleeding is stopped before starting fluid therapy. In addition, a rapid and large volume of fluid administration in trauma patients has been identified as harmful as it could potentially increase the risk of hemorrhage. ${ }^{1,3,7,9,14-16}$ The initial theoretical concept is that aggressive fluid resuscitation increases blood pressure, dislodges early clots, reverses vasoconstriction, and causes coagulopathy by diluting the clotting factors (dilutional coagulopathy), further acidosis, and cellular injury ${ }^{4,17-22}$ (Figure 1); this principle has since been proven through several experimental works and on an uncontrolled hemorrhagic shock model carried out in animal studies. ${ }^{21,23,24}$

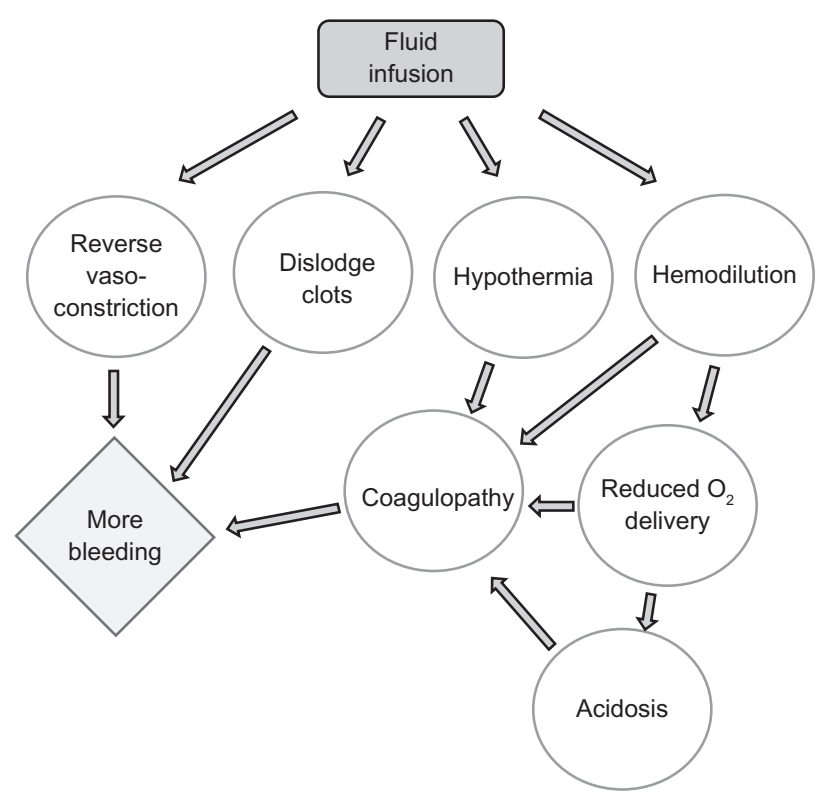

Figure I The impact of aggressive fluid resuscitation on bleeding.
Permissive hypotension has become widely accepted as normal practice in many recommendations and protocols on the basis of strong theoretical grounds and on strong experimental evidence. ${ }^{3,25-30}$ However, many studies and protocols have suggested that cautious fluid resuscitation might not be applicable in blunt trauma, ${ }^{3,6,31}$ although there is no clear evidence to support this. As some authors have argued, there are little data from the studies of human subjects to determine whether blunt injuries behave differently from penetrating injuries..$^{3,25,31}$ With a lack of clear evidence, the burden of proof should fall on the intervention (fluid administration). ${ }^{3}$

The aim of this systematic review is to search for evidence of whether or not the permissive hypotension approach in the management of blunt abdominal trauma patients with hemorrhagic shock but without head or spine injuries or burns is indeed harmful.

\section{Objectives}

The general idea of this study is based on the debate between the "too much too early" fluid resuscitation and "too little too late" fluid administration in managing trauma victims with shock of hemorrhagic origin. However, even with the emerging evidence and the presence of several recommendations and protocols supporting the hypotensive approach, $9,10,18,25,32-34$ there is still reluctance to apply this approach in cases of blunt trauma. This reluctance, in particular, has led to the question addressed in this study: does controlled fluid administration before definitive methods of hemostasis in blunt trauma patients of any age group with hemorrhagic shock, but not with head or spinal injury or burns, improve outcome compared to rapid fluid administration in the same group with the same injury? The focus of this review is to search for evidence of whether rapid intravenous fluid resuscitation (versus controlled fluid administration) has been undertaken in these types of injuries before definitive means of hemostasis, such as surgery, in both prehospital settings and accident and emergency department management.

It is not possible to cover all aspects of trauma management in this review. This review will focus only on phase I (from injury to operation for control of bleeding) ${ }^{1}$ and on blunt abdominal trauma without head or spine injury or burns, which remains one of the most debatable areas in trauma resuscitation. ${ }^{3,6,7,31}$

\section{Method Protocol}

The study design is a systematic review and the methodology is based on the recommended steps provided in the Cochrane 
Handbook for Systematic Reviews of Interventions. ${ }^{35}$ The reporting of the review follows the Preferred Reporting Items for Systematic Reviews and Meta-Analyses Statement and checklist. $^{36}$

\section{Eligibility criteria}

The inclusion of studies follows a flowchart (Figure 2) that is based on clearly predefined inclusion and exclusion criteria, which are as follows.

\section{Inclusion criteria for clinical trials}

Study design: randomized controlled trial (RCT).

Participants/populations: patients with blunt torso injury and hemorrhagic shock of any age group. Trials with a mix of injuries are also included but only the relevant data concerning blunt torso trauma are retrieved. Studies that excluded blunt injuries are reviewed to search for any specific reason as to why blunt injuries were excluded Excluding blunt injuries was not based on evidence, rather, it seemed based on a general trend of reluctancy.

Interventions: rapid or early fluid administration.

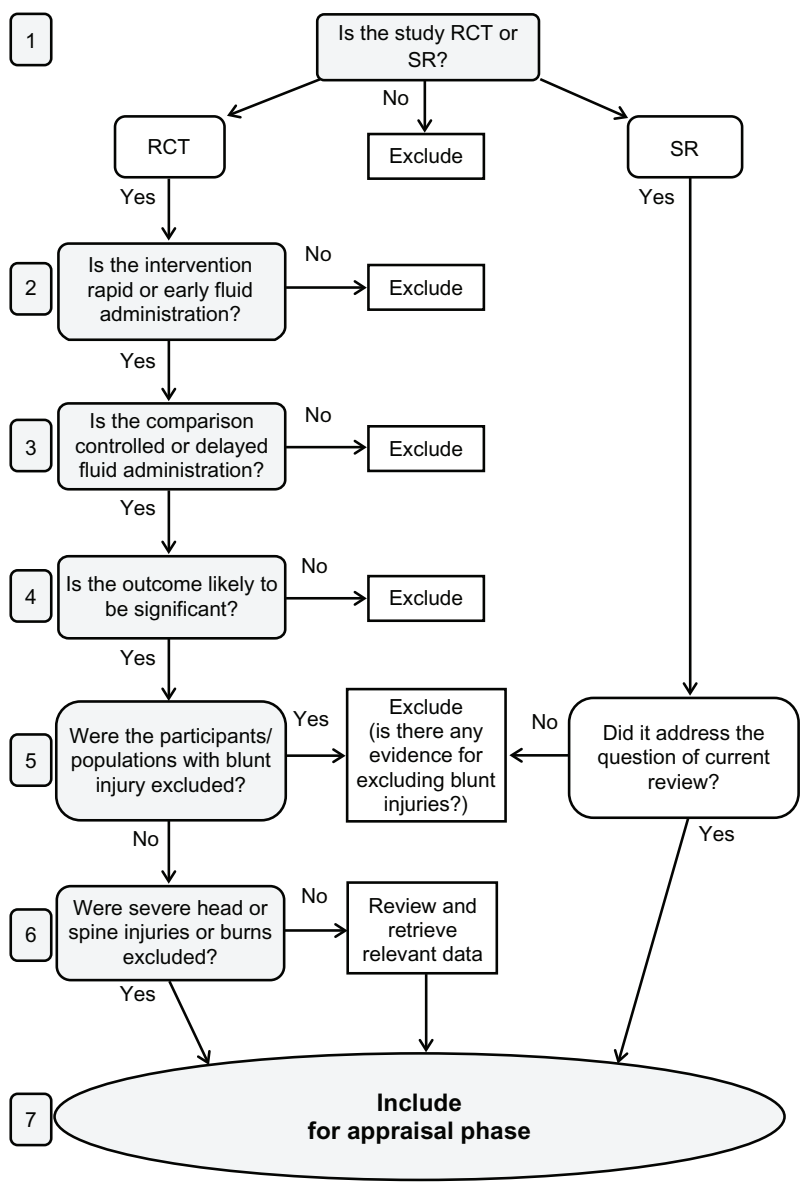

Figure 2 Flowchart for the inclusion of studies. Abbreviations: RCT, randomized controlled trial; SR, systematic review.
Comparisons: controlled or delayed fluid administration.

Outcomes: any outcome that is likely to be meaningful to clinicians, patients, the general public, administrators, and policy makers. This includes morbidity, mortality, and hospital stay.

\section{Inclusion criteria for systematic reviews}

Any systematic review with a primary research question addressing the question of this review.

\section{Exclusion criteria}

Study design: any other study design.

Participants/populations: patients with severe head or spine injury or burn as well as causes of shock other than hemorrhage.

The reason for excluding head or spine injuries is that these injuries cause disturbance of the normal physiology of circulation which interferes with the results. Substantial clinical literature supporting the absolute contraindication of hypotensive resuscitation in traumatic head injury is another reason. ${ }^{37}$ Instances of burns were also not sought due to the different mechanisms and injury management involved.

Interventions/comparisons: studies comparing different types of fluids or comparing blood or blood products to different types of fluids.

Outcomes: trivial outcomes or outcomes that are unlikely to be significant for long-term differences.

\section{Information sources}

Studies were sought by searching internet databases, and by handsearching and contacting authors of previous studies, as well as experts and relevant organizations for any unpublished and ongoing studies. The search for studies adopted a low threshold search strategy and was carried out in six phases (Figure 3) to minimize the risk of loss of relevant papers. Thorough database searches on MEDLINE, EMBASE, the Centre for Reviews and Dissemination, and the Cochrane Library as well as a handsearch were carried out in the first phase and a total of 3357 titles were reviewed. Where the titles seemed relevant, the whole paper was retrieved after the duplicates were removed (phases 2 and 3 ). These papers were reviewed (phase 4) and studies that met the inclusion criteria were examined (phases 5 and 6). The MEDLINE and EMBASE databases were searched using the UK National Health Service Library website. The Centre for Reviews and Dissemination and Cochrane Library databases were searched on their websites. 


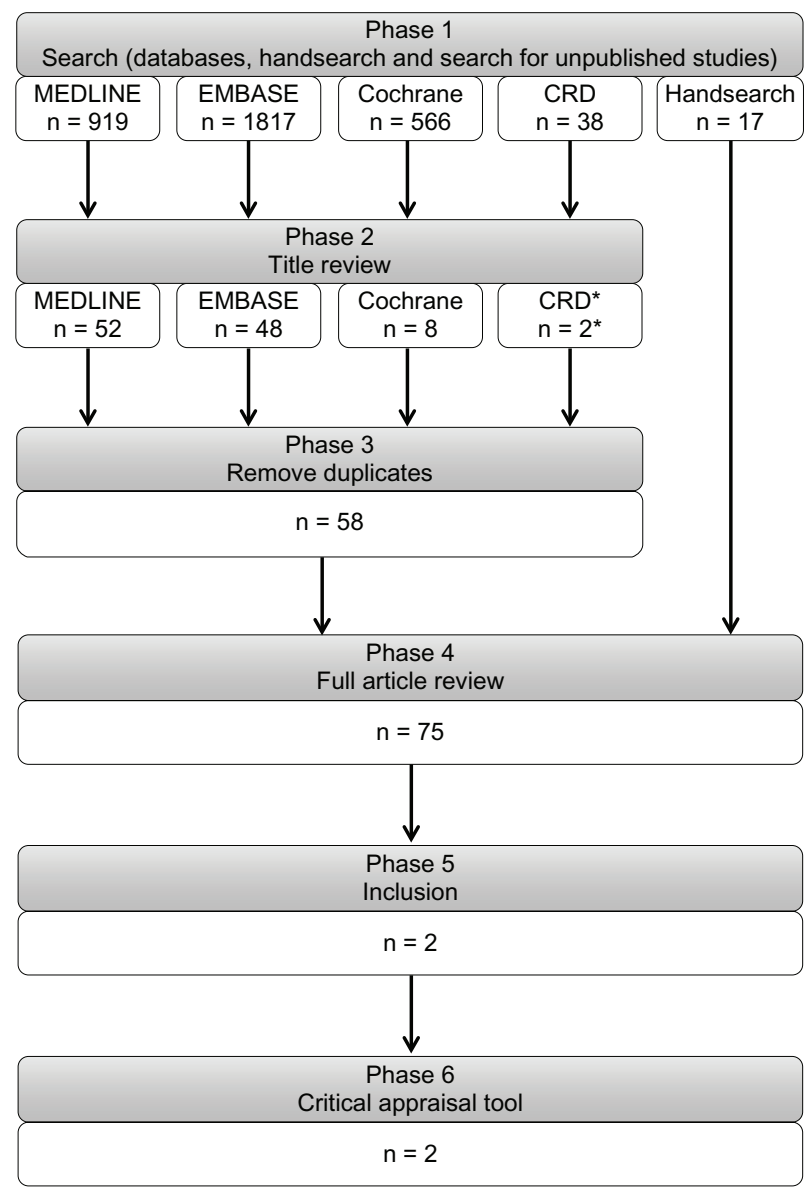

Figure 3 Phases of search for studies.

Abbreviation: CRD, the Centre for Reviews and Dissemination.

The only exclusion criterion during the bibliographic search was animal studies. Article type was not filtered and this, with the adopted strategy of low threshold search, resulted in the return of a very large number of titles. Similarly, retrieving the whole paper when the title seemed relevant resulted in 75 articles being fully reviewed, which, in turn, resulted in the large number of excluded studies and the inclusion of only two trials.

\section{Search}

The following search strategy was used to search the MEDLINE database, and a similar search strategy and similar terminology were used to search the other databases (EMBASE, the Centre for Reviews and Dissemination and the Cochrane Library):

1. trauma*.ti,ab,kw

2. injur*.ti,ab,kw

3. (hemorrhag* OR haemorrhag*) AND shock.mp
4. (hypovolemi* OR hypovolaemi*) AND shock.mp

5. trauma* AND shock.mp

6. "blood loss".mp

7. bleeding.mp

8. (abdo* OR torso OR trunk) AND (injur* OR trauma*).mp

9. spleen AND (injur* OR trauma*).mp

10. liver AND (injur* OR trauma*).mp

11. organ AND (injur* OR trauma*).mp

12. exp "WOUNDS AND INJURIES"/

13. exp SHOCK, HEMORRHAGIC/ OR exp SHOCK, TRAUMATIC/

14. exp HEMORRHAGE/

15. 1 OR 2 OR 3 OR 4 OR 5 OR 6 OR 7 OR 8 OR 9 OR 10 OR 11 OR 12 OR 13 OR 14

16. (fluid OR blood OR volume) AND restor*.mp

17. (fluid OR blood OR volume) AND resuscitat*.mp

18. (fluid OR blood OR volume) AND replac*.mp

19. (fluid OR blood) AND infus*.mp

20. (fluid OR blood) AND administ*.mp

21. "fluid therapy".ti,ab,kw

22. rehydrat*.ti,ab,kw

23. (replace* OR resuscit* OR infus* OR administrat* OR therap*) AND (IV OR intravenous*).mp

24. exp FLUID THERAPY/

25. exp INFUSIONS, INTRAVENOUS/

26. exp BLOOD TRANSFUSION/

27. 16 OR 17 OR 18 OR 19 OR 20 OR 21 OR 22 OR 23 OR 24 OR 25 OR 26

28. restricted AND resuscitation.ti,ab,kw

29. controlled AND hypotension.ti, ab,kw

30. controlled AND resuscitation.ti,ab,kw

31. controlled AND resuscitation.ti,ab,kw

32. delayed AND resuscitation.ti,ab,kw

33. permissive AND hypotension.ti,ab,kw

34. hypotensive AND resuscitation.ti,ab,kw

35. damage AND control AND resuscitation.ti,ab,kw

36. 28 OR 29 OR 30 OR 31 OR 32 OR 33 OR 34 OR 35

37. 15 AND 27 AND 36

38. 37 [Limit to: Humans]

\section{Study selection}

After retrieving the full text of potentially relevant papers, the inclusion and exclusion criteria were applied to meet the aims of this review. To classify papers as RCTs, a flowchart was used to meet the criteria implemented by the US Cochrane Center, as described in the Training Manual for Handsearchers. ${ }^{38}$ 


\section{Data collection}

The data were collected based on the question raised in this review and on the inclusion and exclusion criteria. The collected data were related to blunt trauma injury without head or spine injury and treated with rapid or early fluid administration or controlled or delayed fluid administration.

\section{Risk of bias in individual studies}

Papers that were eligible for inclusion were appraised and the risk of bias in the design and the conduct of the study was assessed. To avoid any risk of bias during the appraisal of the studies, an appraisal checklist tool was used with the permission of the Solutions for Public Health. ${ }^{39}$

\section{Results \\ Study characteristics}

The search did not find any RCTs that specifically studied the rapid versus controlled/delayed fluid resuscitation in blunt trauma. However, two studies, without excluding these injuries, were found. ${ }^{6,37}$

\section{Turner et $\mathrm{al}^{6}$}

This trial compared two protocols of fluid resuscitation in the management of trauma patients on the basis of immediate versus delayed fluid administration. It was carried out in two ambulance-service areas with a mix of urban, suburban, and rural environments. The study was focused on moderate and severe injuries. Paramedics, rather than patients, were randomized (to "Protocol A" and "Protocol B" groups) due to the difficulty of the process. The two ambulance services crossed over protocols halfway through the trial. Throughout the 17 months of the trial, 1309 patients were included retrospectively according to the predefined inclusion and exclusion criteria. The assessed outcomes were mortality, morbidity, intensive care unit admission, change in Triage Revised Trauma Score, and economic evaluation. The latter, however, is beyond the scope of this review.

\section{Dutton et al ${ }^{37}$}

This trial compared fluid resuscitation in the management of trauma with active bleeding and hemorrhagic shock between two targets of systolic blood pressure (SBP) (the "conventional" group received fluid for an SBP of more than $100 \mathrm{mmHg}$ and the "low" group received fluid for an SBP of $70 \mathrm{mmHg}$ ). The study was carried out over 20 months and
110 patients enrolled in the study were randomized according to the inclusion and exclusion criteria. After the end of the period of "active bleeding", management was continued in both groups in line with the ATLS guidelines ${ }^{8}$ and with the protocols of the local trauma center. The recorded outcome was survival to discharge. Follow-up of patients was continued until patient's discharge or death.

\section{Systematic reviews}

The search did not find any systematic review that specifically addressed the question raised by this review.

\section{Risk of bias within studies}

\section{Turner et al $^{6}$}

This trial had a clear aim and the follow-up time was clearly defined. It also had an appropriate study design, which is an RCT. Although the process of randomizing paramedics rather than patients was meant to solve the difficulties of randomization, it only served to add more confusion and difficulties in implementing the inclusion and exclusion criteria. Likewise, it caused a significant crossover between the groups. However, a reasonable effort was made to ensure that all eligible patients were included. Nevertheless, more confusion arose when the inclusion and the exclusion criteria were applied retrospectively to the patients' records. Another cause of concern with regards to the methodology of the trial was the mixing of the selection criteria and the outcome. Admission to the intensive care unit and death were both considered in the inclusion criteria and both were counted as an outcome of assessment. Patients and therapists were not blinded to the study. We were not informed whether or not any effort was made to achieve this. However, the authors argued that "in pragmatic trials, the blinding of patients and therapists may be inappropriate anyway and 'hard' outcomes, such as mortality, are objective and not subject to the influence of personal assessment." Nevertheless, mortality was not the only outcome assessed and one might argue about the validity of this position with respect to the other outcomes and with the questionnaire used for morbidity assessment.

The power calculation was performed and there was no reason to suggest that groups were treated differently or were subjected to different attention. Data analysis was objective and the odds ratio was estimated with a $95 \%$ confidence interval.

An attempt was made to cover wide and varied areas and the outcomes measured in the study were meaningful. 
However, a significant number of records were lost during the study.

\section{Dutton et $\mathrm{al}^{37}$}

The trial addressed a clear question. However, the intervention was not as clear. Although the hypothesis stated that conventional fluid administration ${ }^{37}$ was used as the comparative control, this was not clearly defined. "Conventional" could be understood as the routine ATLS protocol of rapid fluid administration. This confusion was made worse by the fact that sustained SBP that was higher than the target level was managed by the restriction of fluids and by the administration of appropriate doses of anesthetic or analgesic medication. ${ }^{37}$

The study had an appropriate design (RCT) and the population of the study was explained with sufficient clarity. However, it was not clear whether all eligible patients were enrolled. In addition, no details regarding the randomization method of the enrolled patients were given. Similarly, there was no mention of blinding patients to the study. Treating physicians were aware of the inclusion of the patients but did not know the group assignment before the randomization of the patients.

No details were provided about protocol compliance or crossover between groups. However, although the target SBP in the "low" group was $70 \mathrm{mmHg}$ compared to the target of more than $100 \mathrm{mmHg}$ in the "conventional" group, the average SBP was $100 \mathrm{mmHg}$ in the "low" group compared to the average SBP of $114 \mathrm{mmHg}$ in the "conventional" group. Moreover, the use of sedation or analgesia to titrate SBP to the target if indicated created a discrepancy between the methodology and the hypothesis of the study.

Although there was no evidence to indicate whether or not the groups were treated differently, we do not know what specific management each group received during the period of active hemorrhage. There was also no indication as to the number of patients needed to observe a statistically significant difference in the outcome. It is highly unlikely that power calculation was undertaken in this trial. In fact, the authors had indicated that "more than 500 additional patients would have to be enrolled to demonstrate a statistical difference in survival", ${ }^{37}$ and, therefore, a difference between the groups might have emerged if the trial had been continued for a longer time. However, the data were drawn objectively and analyzed appropriately and the $P$ value was reported.

The primary outcome (survival) was significant, but there was no clear cut-off time for the outcome of the study, which was measured on the basis of discharge from hospital. Moreover, there was insufficient explanation of the surrounding environment of included patients. Therefore, it is even harder to generalize the results for all trauma circumstances.

\section{Data analysis}

\section{Turner et al ${ }^{6}$}

Of the 1309 patients enrolled in the study, 699 patients were treated by paramedics operating Protocol A (immediate fluids) while 611 patients were operated by paramedics operating Protocol B (delayed fluids). The baseline of the characteristics between these groups was similar and there was no statistically significant difference in the outcome (Table 1).

Although 98.2\% $(n=1284)$ of the cases were classified as blunt injuries, for the interest of this review, only 469 patients had blunt trunk injury. Out of these 469 patients, 258 were treated under Protocol A and 211 were treated under Protocol B. However, no further data were available with which to compare the subgroups. Furthermore, this trial studied the prehospital phase of pre-definitive control of hemorrhage only (both groups treated similarly in hospital). Therefore, results related to blunt injuries could not be concluded.

\section{Dutton et $\mathrm{al}^{37}$}

One hundred and ten patients were entered into the study. These patients were split into two groups, each group comprising half of the patients. There was no significant difference in the degree of injury or in the outcome between the groups.

The proportion of patients who sustained blunt trauma was $49 \%(n=54)$. However, there were no available data on the specific characteristics of injury in mortality cases. Therefore, further analysis of the blunt trauma subgroup was not possible. Table 2 presents a summary of the data.

As the corresponding author indicated in a personal written communication, there was also no difference on the

Table I Summary of data in Turner et al study ${ }^{6}$

\begin{tabular}{lll}
\hline & Protocol A & Protocol B \\
\hline Total number of patients & 699 & 610 \\
Trauma-related deaths & 58 & 49 \\
Complications & 60 & 46 \\
Admission to ICU & I48 & II3 \\
Number of blunt trunk injuries & 258 & 2 II \\
Deaths in blunt injuries & N/A & N/A \\
Complications in blunt injuries & N/A & N/A \\
Admission to ICU in blunt injuries & N/A & N/A \\
\hline
\end{tabular}

Abbreviations: ICU, intensive care unit; N/A, data not available. 
Table 2 Summary of data in Dutton et al study ${ }^{37}$

\begin{tabular}{lll}
\hline & $\begin{array}{l}\text { Conventional } \\
\text { fluids group }\end{array}$ & $\begin{array}{l}\text { Low fluids } \\
\text { group }\end{array}$ \\
\hline Total number of patients & 55 & 55 \\
Average ISS of all patients & $19.65 \pm \mathrm{II} .84$ & $23.64 \pm 3.82$ \\
Total number of deaths & 4 & 4 \\
Number of blunt injuries & 23 & $3 \mathrm{I}$ \\
Average ISS in blunt injuries & $\mathrm{N} / \mathrm{A}$ & $\mathrm{N} / \mathrm{A}$ \\
Number of deaths in blunt injuries & $\mathrm{N} / \mathrm{A}$ & $\mathrm{N} / \mathrm{A}$ \\
\hline
\end{tabular}

Abbreviations: ISS, Injury Severity Score; N/A, data not available.

blunt or penetrating subgroup analysis (R.P. Dutton, written communication, April 2010).

\section{Discussion}

\section{Summary of evidence}

In view of the high risk of bias and poor methodology, it is unlikely that the results are clinically useful. Therefore, it is hard to positively or negatively relate the result to clinical practice. In addition, conducting a meta-analysis from the available data is not possible as the data are insufficient and the two identified studies had mixed groups of blunt and penetrating injuries with very limited available data on the blunt injury group.

\section{Limitations}

Both studies lack good evidence to support or discourage permissive hypotension in blunt trauma management. Apart from the issues raised earlier about the conduct of the studies as well as the lack of available data and the applicability of the results, there are also severe defects in the assessment of outcomes. Methods such as mean arterial pressure, central venous pressure, and pulmonary artery wedge pressure measurement, can provide more reliable measures than SBP. Studies that consider these measures and/or the more recent pulse oximetry plethysmographic waveform variability, arterial line waveform variability, and pulse pressure variability are necessary to discover more specific answers to the very specific question posed by this review.

\section{Conclusion}

Although permissive hypotension is becoming more accepted and several guidelines, protocols, and clinical papers relating to it have appeared, ${ }^{3,5,25-30,32}$ the uncertainty regarding blunt trauma continues to limit the use of this approach in blunt injuries, although there is no clear reason for this uncertainty. The searches undertaken for this review did not find any good evidence against or in favor of permissive hypotension or controlled fluid resuscitation in blunt torso injuries with hemorrhagic shock. The small amount of available data, however, proved the safety of the permissive hypotensive approach, assuring the interest of researchers in the field and encouraging further work on this important area. In a case-control comparative study, Dula et al have reviewed records of blunt trauma victims with an SBP of $\leq 90 \mathrm{mmHg} .{ }^{40}$ Dula et al also compared the outcomes between those who received $>500 \mathrm{~mL}$ of fluids in pre-hospital settings and those who did not receive any pre-hospital fluids. Seventy-five patients who received $>500 \mathrm{~mL}$ pre-hospital fluids, along with a similar number of patients who did not receive fluids, were chosen according to Injury Severity Score and SBP. The authors did not find a significant difference in the length of hospital stay or survival to discharge between the two groups, although the group who received pre-hospital fluids was more likely to have an increase in SBP upon arrival at the emergency department. ${ }^{40}$ Although a good effort was made by the authors to match the groups and to avoid selection bias, it was not a level one evidence trial. In addition, it included only a small number of patients in each group. Likewise, this trial only studied pre-hospital fluid resuscitation and we do not know whether any extensive resuscitation had been undertaken in the emergency department before the definitive management of hemorrhage. The results of this trial cannot be applied to clinical practice without good quality evidence. However, they should provide reassurance and encouragement to researchers to perform large-scale randomized trials.

The animal model of uncontrolled hemorrhage after massive splenic injury ${ }^{23}$ and the aortotomy model ${ }^{41}$ provided a $^{2}$ conclusion that liberal resuscitation resulted in a significant increase in hemorrhage. Furthermore, the effect of permissive hypotension in improving hemostasis and, consequently, the reduction of blood loss was proved conclusively in a recent trial of aortic injury models in rabbits. ${ }^{42}$ Therefore, the practice of permissive hypotension in trauma patients should be taken into greater consideration. In fact, the concept of hypotensive resuscitation is now the established practice for some nontraumatic causes of hemorrhagic shock. The minimal resuscitation approach for the management of ruptured abdominal aortic aneurism has, in the past several years, been endorsed by many vascular surgeons. ${ }^{29,43,44}$ In the initial fluid resuscitation of bleeding esophageal varices, it is recommended to titrate fluid administration to an SBP of $80-90 \mathrm{mmHg}$, as aggressive fluid resuscitation might increase bleeding. ${ }^{45}$ Moreover, deliberate hypotension has been applied for more than half a century as an anesthetic strategy to minimize blood loss during 
surgery (induced trauma). ${ }^{46,47}$ Therefore, the reluctance and concern about ethical issues related to carrying out further investigations in this important area are not justified. In fact, more exploration and further multicenter quality randomized controlled trials are urgently needed.

\section{Disclosure}

The author reports no conflicts of interest in this work.

\section{References}

1. Revell M, Greaves I, Porter K. Endpoints for fluid resuscitation in hemorrhagic shock. J Trauma. 2003;54(Suppl 5):S63-S67.

2. American College of Surgeons Committee on Trauma. Advanced Trauma Life Support for Doctors ATLS Student Course Manual. 8th ed. Chicago, IL: American College of Surgeons; 2008.

3. Dretzke J, Sandercock J, Bayliss S, Burls A. Clinical effectiveness and cost-effectiveness of prehospital intravenous fluids in trauma patients. Health Technol Assess. 2004;8(23):iii:1-103.

4. Geeraedts LM Jr, Kaasjager HA, van Vugt AB, Frölke JP. Exsanguination in trauma: A review of diagnostics and treatment options. Injury. 2009;40(1):11-20.

5. Kwan I, Bunn F, Roberts I. Timing and volume of fluid administration for patients with bleeding. Cochrane Database Syst Rev. 2003;3:CD002245.

6. Turner J, Nicholl J, Webber L, Cox H, Dixon S, Yates D. A randomised controlled trial of prehospital intravenous fluid replacement therapy in serious trauma. Health Technol Assess. 2000;4(31):1-57.

7. Stern SA. Low-volume fluid resuscitation for presumed hemorrhagic shock: helpful or harmful? Curr Opin Crit Care. 2001;7(6):422-430.

8. American College of Surgeons Committee on Trauma. Advanced Trauma Life Support for Doctors ATLS Student Course Manual. 7th ed. Chicago, IL: American College of Surgeons; 2004.

9. Berry RD. Management of shock in trauma. Anaesth Intensive Care Med. 2008;9(9):390-393.

10. MacKinnon MA. Permissive hypotension: A change in thinking. Air Med J. 2005;24(2):70-72.

11. Hamiliton SM, Breakey P. Fluid resuscitation of the trauma patient: how much is enough? Can J Surg. 1996;39(1):11-16.

12. Bagshaw SM, Bellomo R. The influence of volume management on outcome. Curr Opin Crit Care. 2007;13(5):541-548.

13. Tintinalli JE, Kelen GD, Stapczynski JS. Emergency Medicine: A Comprehensive Study Guide. 6th ed. New York, NY: McGraw-Hill; 2003.

14. Roberts I, Evans P, Bunn F, Kwan I, Crowhurst E. Is the normalisation of blood pressure in bleeding trauma patients harmful? Lancet. 2001;357(9253):385-387.

15. Mattox KL, Brundage SI, Hirshberg A. Initial resuscitation. New Horiz. 1999;7(1):4-9.

16. Nolan J. Fluid resuscitation for the trauma patient. Resuscitation. 2001;48(1):57-69.

17. Alam HB. An update on fluid resuscitation. Scand J Surg. 2006;95(3): 136-145.

18. Bickell WH, Wall MJ Jr, Pepe PE, et al. Immediate versus delayed fluid resuscitation for hypotensive patients with penetrating torso injuries. N Engl J Med. 1994;331(17):1105-1109.

19. Leppäniemi A, Soltero R, Burris D, et al. Fluid resuscitation in a mode of uncontrolled hemorrhage: too much too early or too little too late? J Surg Res. 1996;63(2):413-418.

20. Selby JB, Mathis JE, Berry CF, Hagedorn FN, Illner HP, Shires GT. Effect of isotonic saline solution resuscitation on blood coagulation in uncontrolled hemorrhage. Surgery. 1996;119(5):528-533.
21. Sondeen JL, Coppes VG, Holcomb JB. Blood pressure at which rebleeding occurs after resuscitation in swine with aortic injury. J Trauma. 2003;54(Suppl 5):S110-S117.

22. Rotondo MF, Schwab CW, McGonigal MD, et al. "Damage Control": An approach for improved survival in exsanguinating penetrating abdominal injury. J Trauma. 1993;35(3):375-382.

23. Solomonov E, Hirsh M, Yahiya A, Krausz MM. The effect of vigorous fluid resuscitation in uncontrolled hemorrhagic shock after massive splenic injury. Crit Care Med. 2000;28(3):749-754.

24. Krausz MM, Basheuko Y, Hirsh M. Crystalloid or colloid resuscitation of uncontrolled hemorrhagic shock after moderate splenic injury. Shock. 2000;13(3):230-235.

25. Greaves I, Porter KM, Revell MP. Fluid resuscitation in pre-hospital trauma care: a consensus view. J R Coll Surg Edinb. 2002;47(2):451-457.

26. Nolan J. Fluid replacement. Br Med Bull. 1999;55(4):821-843.

27. Shirley PJ, Weaver AE. Hypotensive resuscitation in trauma. J Trauma. 2002;53(6):1196.

28. Holmes DF, Sakles JC, Lewis G, Wisner DH. Effects of delaying fluid resuscitation on an injury to the systematic arterial vasculature. Acad Emerg Med. 2002;9(4):267-274.

29. Myers C. Fluid resuscitation. Eur J Emerg Med. 1997;4(4):224-232.

30. Driscoll P, Kent A. The effect of scene time on survival. Trauma. 1999;1(1):23-30.

31. Levett D, Vercueil A, Grocott M. Resuscitation fluids in trauma 1: why give fluid and how to give it. Trauma. 2006;8(1):47-53.

32. Hai SA. Permissive hypotensive resuscitation - An evolving concept in trauma. J Pak Med Assoc. 2004;54(8):434-436.

33. Spahn DR, Cerny V, Coats TJ, et al; for the Task Force for Advanced Bleeding Care in Trauma. Management of bleeding following major trauma: a European guideline. Crit Care. 2007;11(1):R17.

34. Rossaint R, Bouillon B, Cerny V, et al; for the Task Force for Advanced Bleeding Care in Trauma. Management of bleeding following major trauma: an updated European guideline. Crit Care. 2010; 14(2):R52.

35. Higgins JPT, Green S, editors. Cochrane Handbook for Systematic Reviews of Interventions. Version 5.1.0 [updated Mar 2011]. The Cochrane Collaboration; 2009. Available from: http://www.cochranehandbook.org. Accessed April 9, 2012.

36. Moher D, Liberati A, Tetzlaff J, Altman DG; for the PRISMA Group. Preferred Reporting Items for Systematic Reviews and Meta-Analyses: The PRISMA Statement. Open Med. 2009;3(3):123-130.

37. Dutton RP, Mackenzie CF, Scalea TM. Hypotensive resuscitation during active hemorrhage: impact on in-hospital mortality. J Trauma. 2002;52(6):1141-1146.

38. US Cochrane Center. Training Manual for Handsearchers. The Cochrane Collaboration; 2002. Available from: http://us.cochrane.org/ sites/us.cochrane.org/files/uploads/pdf/Handsearcher\%20Training\%20 Manual.pdf. Accessed April 9, 2012.

39. Critical Appraisal Skills Programme (CASP). 10 Questions to Help You Make Sense of Qualitative Research. England: Public Health Resource Unit; 2006. Available from: http://www.sph.nhs.uk/sph-files/ casp-appraisal-tools/Qualitative\%20Appraisal\%20Tool.pdf. Accessed April 9, 2012

40. Dula DJ, Wood GC, Rejmer AR, Starr M, Leicht M. Use of prehospital fluids in hypotensive blunt trauma patients. Prehosp Emerg Care. 2002;6(4):417-420.

41. Owens TM, Watson WC, Prough DS, Uchida T, Kramer GC. Limiting initial resuscitation of uncontrolled hemorrhage reduces internal bleeding and subsequent volume requirements. J Trauma. 1995;39(2):200-207.

42. Rezende-Neto JB, Rizoli SB, Andrade MV, et al. Permissive hypotension and desmopressin enhance clot formation. J Trauma. 2010;68(1):42-50.

43. Crawford ES. Ruptured abdominal aortic aneurysm J Vasc Surg. 1991;13(2):348-350. 
44. Johansen K, Kohler TR, Nicholls SC, Zierler RE, Clowes AW, Kazmers A. Ruptured abdominal aortic aneurysm: the Harborview experience. J Vasc Surg. 1991;13(2):240-245.

45. Gow PJ, Chapman RW. Modern management of oesophageal varices. Postgrad Med J. 2001;77(904):75-81.
46. Brett AS. Fluid resuscitation in the initial management of post-traumatic shock: the concept of permissive hypotension. Clinical Intensive Care. 2000;11(3):121-126.

47. Eckenhoff JE, Rich JC. Clinical experiences with deliberate hypotension. Anesth Analg. 1966;45(1):21-28.

Open Access Emergency Medicine

\section{Publish your work in this journal}

Open Access Emergency Medicine is an international, peer-reviewed, open access journal publishing original research, reports, editorials, reviews and commentaries on all aspects of emergency medicine. The manuscript management system is completely online and includes a very quick and fair peer-review system, which is all easy to use.

\section{Dovepress}

Visit http://www.dovepress.com/testimonials.php to read real quotes from published authors. 